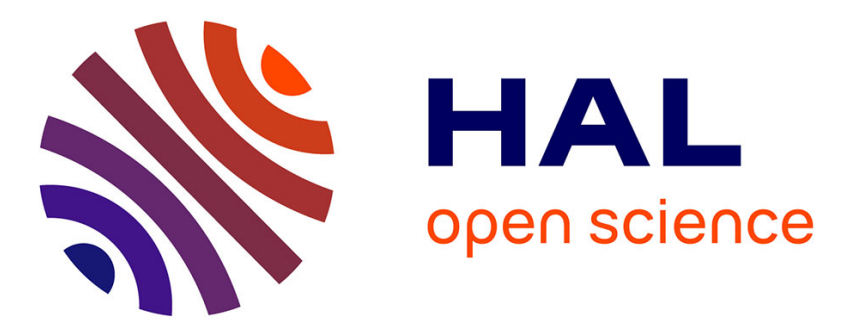

\title{
A real-time and robust intrusion detection system with commodity wi-fi
}

Shengjie Li, Zhaopeng Liu, Yue Zhang, Xiaopeng Niu, Leye Wang, Daqing Zhang

\section{- To cite this version:}

Shengjie Li, Zhaopeng Liu, Yue Zhang, Xiaopeng Niu, Leye Wang, et al.. A real-time and robust intrusion detection system with commodity wi-fi. UbiComp/ISWC 2019 Adjunct: 2019 ACM international joint conference on Pervasive and Ubiquitous Computing and International Symposium on Wearable Computers, Sep 2019, London, United Kingdom. pp.316-319, 10.1145/3341162.3343789 . hal-03002793

\section{HAL Id: hal-03002793 https://hal.science/hal-03002793}

Submitted on 12 Nov 2020

HAL is a multi-disciplinary open access archive for the deposit and dissemination of scientific research documents, whether they are published or not. The documents may come from teaching and research institutions in France or abroad, or from public or private research centers.
L'archive ouverte pluridisciplinaire HAL, est destinée au dépôt et à la diffusion de documents scientifiques de niveau recherche, publiés ou non, émanant des établissements d'enseignement et de recherche français ou étrangers, des laboratoires publics ou privés. 


\section{Demo: A Real-time and Robust Intrusion Detection System with Commodity Wi-Fi}

\author{
Shengjie Li \\ Peking University \\ Peking University Information \\ Technology Institute (Tianjin Binhai) \\ Beijing, China \\ lishengjie@pku.edu.cn \\ Xiaopeng Niu \\ Peking University \\ Peking University Information \\ Technology Institute (Tianjin Binhai) \\ Beijing, China \\ nxpeng@pku.edu.cn
}

\author{
Zhaopeng Liu \\ Peking University \\ Peking University Information \\ Technology Institute (Tianjin Binhai) \\ Beijing, China \\ liuzp@pku.edu.cn \\ Leye Wang \\ Peking University \\ Beijing, China \\ leyewang@pku.edu.cn
}

\author{
Yue Zhang \\ Peking University \\ Peking University Information \\ Technology Institute (Tianjin Binhai) \\ Beijing, China \\ zy.zhang@pku.edu.cn \\ Daqing Zhang \\ Peking University, Beijing \\ Telecom SudParis, Evry \\ China/France \\ dqzhang@sei.pku.edu.cn
}

\begin{abstract}
Intrusion detection plays a rather important role in many applications, like asset protection and elder caring. Since we cannot make any requirements on the intruder, a device-free passive way of intrusion detection is much more promising and practical. In order to achieve robust passive intrusion detection, various techniques have been proposed, including video-based, infra-based and sensor-based approaches, among which dedicated device installation is often required. In this work, we present a real-time and robust device-free intrusion detection system, named RR-Alarm. By reusing the existing Wi-Fi signals, RR-Alarm is able to detect human intrusion in real time, at the same time, requiring no additional facilities installation. By utilizing the Doppler effects incurred by human motion on multiple Wi-Fi devices, RR-Alarm is not only able to accurately detect the intrusion without any extra human efforts but also avoids a large number of false alarms caused by the human motion from outside the house. A long-term trial in a nursing home verifies the effectiveness of our Wi-Fi based RR-Alarm system.
\end{abstract}

\section{CCS CONCEPTS}

- Human-centered computing $\rightarrow$ Ubiquitous and mobile computing systems and tools.

Permission to make digital or hard copies of part or all of this work for personal or classroom use is granted without fee provided that copies are not made or distributed for profit or commercial advantage and that copies bear this notice and the full citation on the first page. Copyrights for thirdparty components of this work must be honored. For all other uses, contact the owner/author(s).

UbiComp/ISWC '19 Adjunct, September 9-13, 2019, London, United Kingdom (c) 2019 Copyright held by the owner/author(s).

ACM ISBN 978-1-4503-6869-8/19/09.

https://doi.org/10.1145/3341162.3343789

\section{KEYWORDS}

Wi-Fi; Channel State Information (CSI); Intrusion Detection; Device-free Sensing

ACM Reference Format:

Shengjie Li, Zhaopeng Liu, Yue Zhang, Xiaopeng Niu, Leye Wang, and Daqing Zhang. 2019. Demo: A Real-time and Robust Intrusion Detection System with Commodity Wi-Fi. In Adjunct Proceedings of the 2019 ACM International foint Conference on Pervasive and Ubiquitous Computing and the 2019 International Symposium on Wearable Computers (UbiComp/ISWC '19 Adjunct), September 9-13, 2019, London, United Kingdom. ACM, New York, NY, USA, 4 pages. https://doi.org/10.1145/3341162.3343789

\section{INTRODUCTION}

Device-free intrusion detection tries to tell the existence of the intruder in the area of interest without any device attached to the intruder. It is essential to various smart home applications such as asset protection, home security, child and elder care. In order to achieve device-free intrusion detection, various techniques have been studied and proposed. One of the most popular methods is video-based approach [1] that utilize cameras installed in the environment to capture the image or video sequences for scene recognition. However, such the approach may raise privacy concern and is restricted by lighting condition. Sensor-based approaches [3, 9, 10] make use of information caused by human walking to detect intrusion but require installation of dedicated sensors in the environment.

In recent years, with the rapid development of wireless technologies, the role of Wi-Fi Radio Frequency(RF) signals has been extended from that of a sole communication medium to a non-intrusive environmental sensing tool. We have seen a lot of emerging Wi-Fi based human sensing 
applications, ranging from fine-gained respiration monitoring [12] to coarse-grained activity recognition [4, 13, 15] , indoor localization/tracking [6-8]. As one of the most critical applications, intrusion detection also attracts lots of attention because it is able to provide valuable human state information. Multiple efforts have been made for intrusion/motion detection leveraging channel state information (CSI) available at commodity Wi-Fi devices $[5,11,14]$. These works detect whether a human is still or moving based on the variance or correlation of CSI amplitude or phase difference information in the time domain. As these time-domain signal features are environment-dependent [5], once there are environment changes(i.e., furniture movement), these methods require a significant amount of human efforts in off-line training and calibration to relearn the difference between static environment (i.e., the human is still) and dynamic environment (i.e., the human is non-still). Besides the cumbersome learning process, it is also intrinsically challenging for these systems to avoid false alarms caused by the human motion from outside the house (i.e., hallway walking), which could also result in a significant variation on CSI amplitude and phase difference signal.

Aiming to overcome the limitations mentioned above of previous approaches, in this work, we propose a CSI Doppler effect based human intrusion detection system, called $R R$ Alarm. By understanding indoor signal-propagation properties, RR-Alarm could not only capture the human motion inside the house but also avoids the interference outside the house without any human intervention. The key insight behind it is that in an indoor environment, Wi-Fi signals not only propagate along the direct path to the receiver but also are reflected by objects such as walls, furniture and the human body. The signal at the receiver is the superposition of signals from all paths. When a human target is moving, the human motion will change the path length of the human reflected signal and then introduce a Doppler frequency shift on the Wi-Fi signal. However, other path signals reflected by the static furniture and walls remain unchanged and thus make no contribution to the Doppler frequency shift at the receiver. By capturing this Doppler frequency shift, the human motion can be clearly detected and not affected by other complex multi-path reflected signals in the environment despite potential environmental changes. Another important observation is that when there is a wall blocking the reflected path between the human body and Wi-Fi transceivers, the strength of Doppler frequency shift obtained from Wi-Fi signal will be dramatically weakened. Utilizing this observation, RR-Alarm is able to eliminate the interference of human motion outside the house and achieves a low false alarms rate. In summary, RR-Alarm makes the following contributions: 1) To the best of our knowledge, RR-Alarm is the first Doppler effect based intrusion detection system which

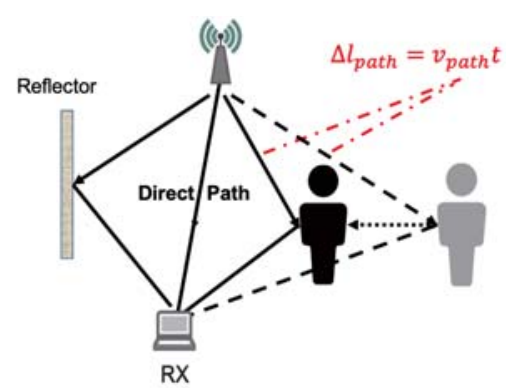

Figure 1: Doppler effect on Wi-Fi signal.

does not require any human intervention by extracting the environment-independent Doppler frequency shift feature on multiple Wi-Fi devices. 2) RR-Alarm eliminates the interference of the human motion outside the house and achieves a very low false alarm rate by utilizing the significant difference of Doppler frequency shift strength between the human motion inside the house and outside the house. 3) RR-Alarm evaluates its effectiveness and reliability over a long-term trial in a nursing home.

\section{METHODS}

To achieve the intrusion detection goal by leveraging surrounding Wi-Fi signal, RR-Alarm makes use of the fundamental physical phenomena called Doppler Effect. Given a pair of Wi-Fi transmitter (TX) and receiver (RX) as shown in Figure 1, the Wi-Fi signal can propagate from the transmitter to the receiver via a direct path and paths reflected by human body, wall and other objects. The human motion would change the path length of the human reflection signal, and thus introduce a Doppler frequency shift to the received signal:

$$
f_{\text {Doppler }}=\frac{f * v_{\text {path }}}{c}
$$

Where $f$ is the carrier frequency of the Wi-Fi signal, $v_{\text {path }}$ is the speed of path length change, and $c$ is light speed. The Doppler frequency shift can be reflected in the CSI (Channel State Information) of the Wi-Fi signal. The CSI represents the amplitude attenuation and phase change of a Wi-Fi signal in each subcarrier, which is incurred by the signal propagation from the transmitter to the receiver. Considering only one signal, its CSI at time $t_{0}$ is $x\left(f, t_{0}\right)=A_{0} e^{-j 2 \pi f \tau_{0}}$, where $A_{0}$ is the attenuation and $\tau_{0}$ is the propagation delay. If the propagation path length changes at a speed of $v$, after a short time period $t$, the path length change $\Delta l_{\text {path }}=v t$ and the propagation delay change $\Delta \tau=\frac{v t}{c}$. When ignoring the attenuation change, the signal's CSI is $x\left(f, t_{0}+t\right)=$ $A_{0} e^{-j 2 \pi f\left(\tau_{0}+\frac{v t}{c}\right)}=x\left(f, t_{0}\right) e^{-j 2 \pi f \frac{v t}{c}}$. The changing frequency of CSI represents the Doppler frequency shift of the signal. For the multi-path propagation scenario in the real world, 


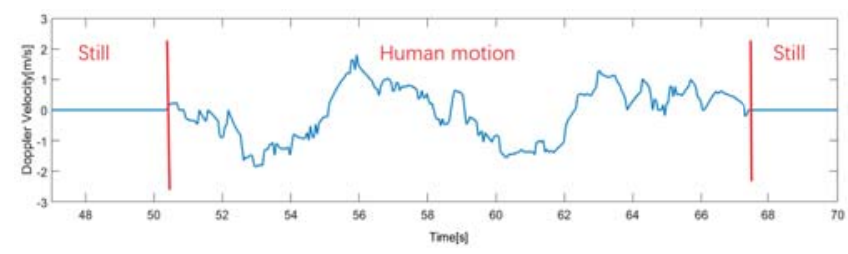

Figure 2: Doppler Velocity of still and moving cases.



Figure 3: Doppler effect in through wall scenario.

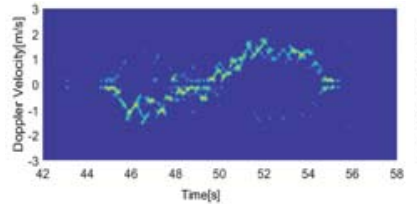

(a) Inside motion

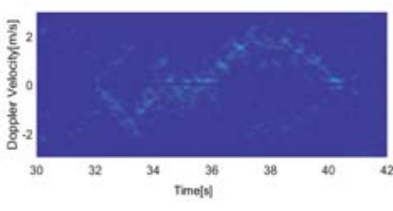

(b) Outside motion
Figure 4: Doppler velocity spectrum when human motion occurs inside and outside the house

the CSI of each packet can be represented as follows:

$$
x\left(f, t_{0}+t\right)=\sum_{i=1}^{L} A_{i} e^{-j 2 \pi f\left(\tau_{i}+\frac{v_{i} t}{c}\right)}
$$

where $L$ is the number of propagation paths, $\tau_{i}$ is the propagation delay of the $i^{t h}$ path signal at time $t_{0}$, and $v_{i}$ is the $i^{t h}$ path's length change speed. Based on Equation 2, we can estimate the Doppler frequency shift introduced by the human motion with DopplerMUISC method [7]. Then based on Equation 1, we could further estimate the path length change speed which is also referred as Doppler velocity. When a human moves in the sensing area, he will change the reflected path length and introduce a non-zero Doppler velocity on Wi-Fi signal as shown in Figure 2. So we are able to utilize Doppler velocity to detect the human motion.

However, for the goal of intrusion detection, detecting human motion is not enough. As Wi-Fi signals transmit ominidirectionally in the space, some Wi-Fi signals will transmit through the wall, reflected by the human body, then finally arrive at the receiver side as shown in Figure 3.

In this situation, the human motion outside the house(i.e., hallway walking) will also introduce a non-zero Doppler velocity on the Wi-Fi CSI which will result in a large number of



Figure 5: Sensing area of different Wi-Fi devices Table 1: Intrusion results combined from two devices

\begin{tabular}{|c|c|c|}
\hline RX1 & RX2 & Result \\
\hline 0 & 0 & Non-intrusion \\
0 & 1 & Intrusion \\
1 & 0 & Intrusion \\
1 & 1 & Intrusion \\
\hline
\end{tabular}

false alarms for human intrusion detection. In order to avoid such kinds of false alarms, we take advantage of the strength of Doppler velocity. Figure 4 shows the Doppler velocity spectrum when human motion occurs inside the house and outside the house where respectively corresponding to the cases of a direct human reflected path and a wall-blocked reflected path. The brightness of Doppler velocity on spectrum indicates the strength of Doppler velocity. As we can see, when there is a wall blocking the reflected path between human body and Wi-Fi transceivers, the strength of Doppler velocity will be dramatically weakened. In other words, only when the human truly enters the room, human reflected path would not be blocked by walls and result in an effective Doppler velocity. Therefore, by extracting the power of the strongest Doppler velocity on spectrum, we could further determine whether human motion occurs outside the house or inside the house, where only human motion within the house means a true intrusion event. Considering that there may exist interior walls(i.e. toilet wall in Figure 5) in a typical house, one single device may have its limited sensing area as shown in Figure 5. Then in order to cover the entire room, we further combine the detection results of two Wi-Fi devices deployed in different places. As shown in Table 1, once there is one device detecting an effective Doppler velocity, it means that an intrusion event has occurred.

\section{DEMONSTRATION SET-UP}

The RRAlram system requires one Wi-Fi transmitter and two Wi-Fi receivers. We use three GIGABYTE miniPCs equipped with off-the-shelf Intel $5300 \mathrm{Wi}$-Fi cards as the transmitter and receiver. The deployment of these three devices in the nursing home is shown as Figure 6.The CSI tool [2] developed by Halperin is installed on the miniPCs to collect CSI for each received packet. We use the $5 \mathrm{GHz}$ frequency band with a $20 \mathrm{MHz}$ channel. In order to capture all Doppler frequency shifts incurred by human motion, the sampling rate of CSI 


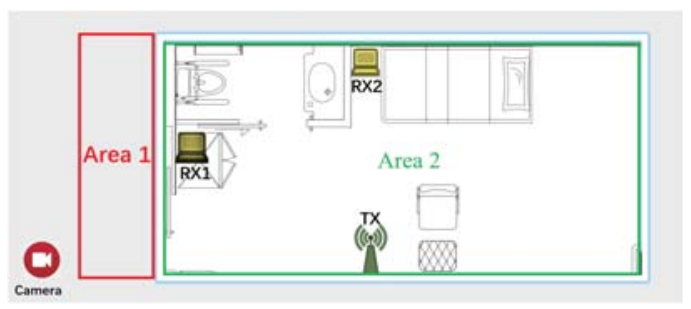

Figure 6: System deployment and monitoring areas division and in the nursing home.

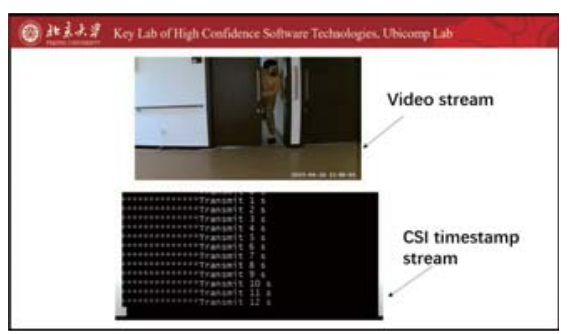

Figure 7: Video frame for video and CSI synchronization.

in RRAlram is set to $200 \mathrm{~Hz}$. RR-Alram makes 20 times of Doppler velocity estimation per second, and each time of Doppler velocity estimation is calculated using CSI samples collected within a time window of 0.3 seconds.

\section{DATASET}

As shown in Figure 6, we divide the monitoring area into two parts: the inner part of the house as Area 1, and the outer part of the house as Area 2.The dataset is organized into five categories: 1)Silence data: No one is in any of the two areas;2)outside-motion data: one or more people are walking in Area 2; 3)inside-motion data: There is only one person walking or doing daily activities in Area 1; 4)Out2In data: with one person walking from Area2 to Area 1. 5)In2Out data: with one person walking from Area1 to Area 2.

A web camera is deployed outside the house and shoot towards the door to record the ground truth. In order to synchronize the video with the CSI, we use a screen-recorder to record the video stream together with the CSI timestamp stream simultaneously. A video frame recorded by the screenrecorder is shown as Figure 7. For each piece of CSI data, there is a corresponding video file. The datasets and ground truth videos are available at our Wi-Fi sensing website ${ }^{1}$. During the long-term deployment in the nursing home, RRAlarm achieves a high intrusion detection rate with an accuracy of $99 \%$ and also holds a low false alarm rate of $0.5 \%$.

\section{ACKNOWLEDGMENTS}

We would like to thank volunteers for their participation and helpful suggestions in this work. The work is supported by the NSFC Grant No.61572048 and Peking University Information Technology Institute (Tianjin Binhai).

\section{REFERENCES}

[1] Q. Cai and J. K. Aggarwal. 1998. Automatic tracking of human motion in indoor scenes across multiple synchronized video streams. In International Conference on Computer Vision. 356-362.

[2] Daniel Halperin, Wenjun Hu, Anmol Sheth, and David Wetherall. 2011. Tool release: Gathering $802.11 \mathrm{n}$ traces with channel state information. ACM SIGCOMM Computer Communication Review 41, 1 (2011), 53-53.

[3] Satish G. Iyengar, Pramod K. Varshney, and Thyagaraju Damarla. 2007. On the Detection of Footsteps Based on Acoustic and Seismic Sensing. In Asilomar Conference on. 2248-2252.

[4] Shengjie Li, Xiang Li, Qin Lv, and Daqing Zhang. 2018. WiFit: A Bodyweight Exercise Monitoring System with Commodity Wi-Fi. 396399. https://doi.org/10.1145/3267305.3267563

[5] Shengjie Li, Xiang Li, Kai Niu, Hao Wang, Yue Zhang, and Daqing Zhang. 2017. AR-Alarm: An Adaptive and Robust Intrusion Detection System Leveraging CSI from Commodity Wi-Fi. 211-223.

[6] Xiang Li, Shengjie Li, Daqing Zhang, Jie Xiong, Yasha Wang, and Hong Mei. 2016. Dynamic-music: accurate device-free indoor localization. In Proceedings of the 2016 ACM International foint Conference on Pervasive and Ubiquitous Computing. ACM, 196-207.

[7] Xiang Li, Daqing Zhang, Qin Lv, Jie Xiong, Shengjie Li, Yue Zhang, and Hong Mei. 2017. IndoTrack: Device-free indoor human tracking with commodity Wi-Fi. Proceedings of the ACM on Interactive, Mobile, Wearable and Ubiquitous Technologies 1, 3 (2017), 72.

[8] Xiang Li, Daqing Zhang, Jie Xiong, Yue Zhang, Shengjie Li, Yasha Wang, and Hong Mei. 2018. Training-Free Human Vitality Monitoring Using Commodity Wi-Fi Devices. Proceedings of the ACM on Interactive, Mobile, Wearable and Ubiquitous Technologies 2, 3 (2018), 121.

[9] Leibo Liu, Weilong Zhang, Chenchen Deng, Shouyi Yin, and Shaojun Wei. 2015. BriGuard: a lightweight indoor intrusion detection system based on infrared light spot displacement. Iet Science Measurement Technology 9, 3 (2015), 306-314.

[10] Robert J Orr and Gregory D Abowd. 2000. The smart floor: a mechanism for natural user identification and tracking. In CHI '00 Extended Abstracts on Human Factors in Computing Systems. 275-276.

[11] Kun Qian, Chenshu Wu, Zheng Yang, Yunhao Liu, and Zimu Zhou. 2014. PADS: Passive detection of moving targets with dynamic speed using PHY layer information. In IEEE International Conference on Parallel and Distributed Systems. 1-8.

[12] Hao Wang, Daqing Zhang, Junyi Ma, Yasha Wang, Yuxiang Wang, Dan $\mathrm{Wu}$, Tao Gu, and Bing Xie. 2016. Human respiration detection with commodity wifi devices: do user location and body orientation matter?. In Proceedings of the 2016 ACM International foint Conference on Pervasive and Ubiquitous Computing. ACM, 25-36.

[13] Hao Wang, Daqing Zhang, Yasha Wang, Junyi Ma, Yuxiang Wang, and Shengjie Li. 2017. RT-Fall: A Real-Time and Contactless Fall Detection System with Commodity WiFi Devices. IEEE Trans. Mob. Comput. 16, 2 (2017), 511-526.

[14] Chenshu Wu, Zheng Yang, Zimu Zhou, Xuefeng Liu, Yunhao Liu, and Jiannong Cao. 2015. Non-Invasive Detection of Moving and Stationary Human With WiFi. IEEE Journal on Selected Areas in Communications 33 (11 2015), 1-1. https://doi.org/10.1109/JSAC.2015.2430294

[15] Daqing Zhang, Hao Wang, and Dan Wu. 2017. Toward centimeterscale human activity sensing with Wi-Fi signals. Computer 50, 1 (2017), 48-57.

\footnotetext{
${ }^{1}$ http://www.pku-wireless-sensing.site/WiLife/
} 